\title{
Assessment of Prediction Algorithms for Ranking Objects
}

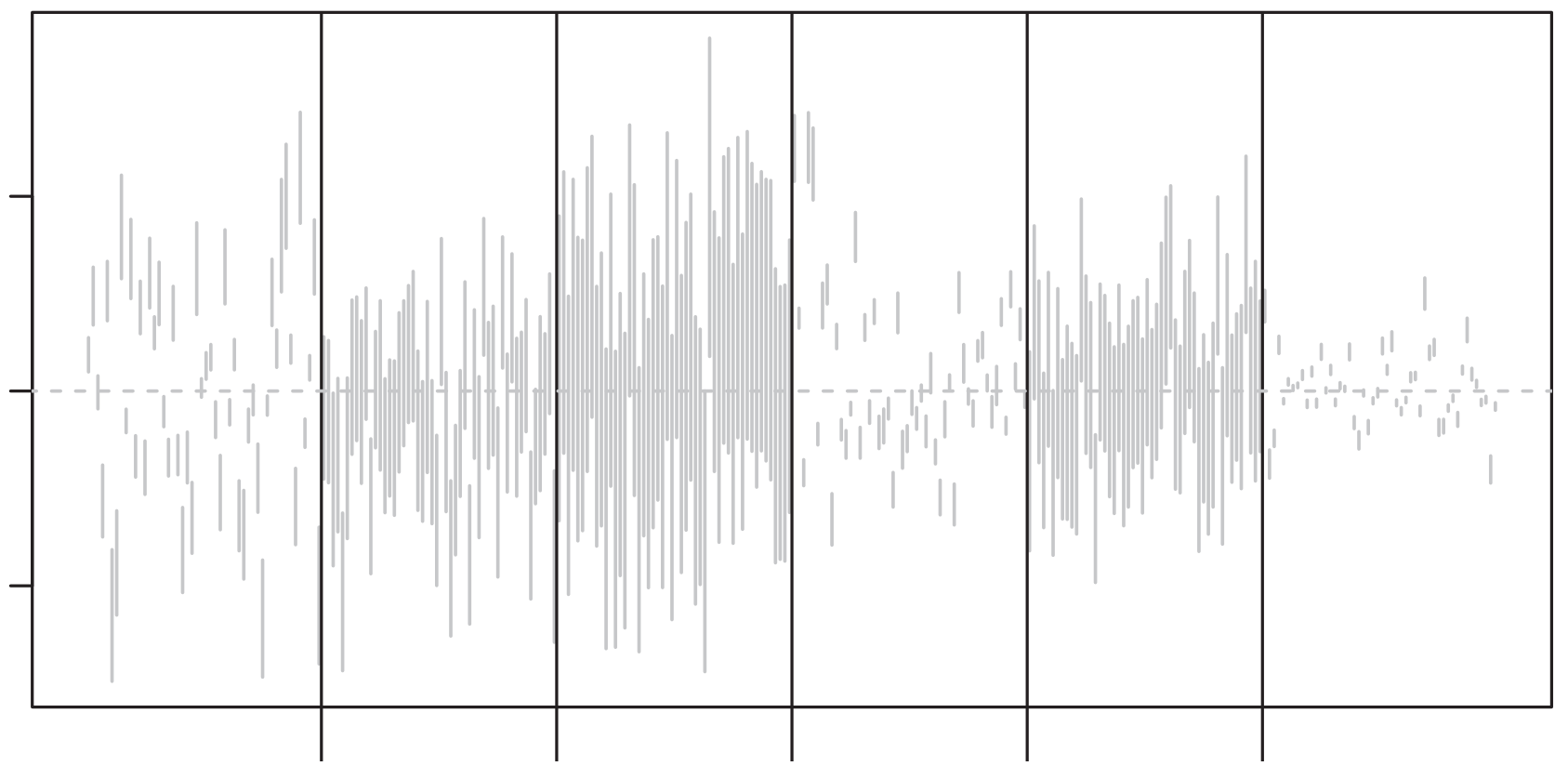

\section{Jacqueline M. Hughes-Oliver}

Prediction algorithms are everywhere [11]. Based on known or observable features of a set of objects, these algorithms provide informed guesses about unknown or unobserved outcomes of the same set of objects. For this article, interest is limited to a single unknown outcome that can take one of two states: positive or negative. In the context of credit scoring, positive may indicate that an applicant for a loan is deemed likely to default on the loan. In the context of screening biomarkers, positive may indicate that a biomarker is strongly associated with a particular disease. In the context of information retrieval, positive may indicate that the returned item is relevant for the request. And in the context of drug discovery in the pharmaceutical industry, positive may indicate that a compound has scored well in a biological assay and has been cleared for followup testing as an active or hit compound. For these and

Jacqueline M. Hughes-Oliver is professor of statistics at North Carolina State University. Her email address is hugheso1@ncsu.edu. This author's ORCID iD is https://orcid.org/0000-0002-6464-9955.

Communicated by Notices Associate Editor Noah Simon.

For permission to reprint this article, please contact:

reprint-permission@ams .org.

DOI: https://doi.org/10.1090/noti1790 other applications, prediction algorithms typically provide a score $(S)$ that represents the belief that an object is in the positive class; this score is based on attributes of the object. Without loss of generality, assume that larger scores indicate strong belief for the positive class.

Prediction algorithms for qualitative or binary outcomes are sometimes referred to as classification algorithms. By applying a threshold $(t)$ to the score, $S>t$ may be used to classify the object as a positive, while $S \leq t$ classifies the object as a negative. On the other hand, the task of ranking goes beyond the task of classification. Prediction algorithms are referred to as ranking algorithms when the returned scores are used to provide an ordered arrangement of objects. Consider an example in credit scoring where five applicants receive scores of 0.9, 0.2, 0.8, 0.4, and 0.7 , respectively. Using a threshold of 0.5 , applicants one, three, and five are classified as likely to default on the loan, while applicants two and four are classified as likely to repay the loan. Classification offers equal treatment to applicants two and four, because we believe they will both repay. Ranking, on the other hand, regards customer two as less risky due to their lower score. If money were available to fund only one applicant, ranking suggests 

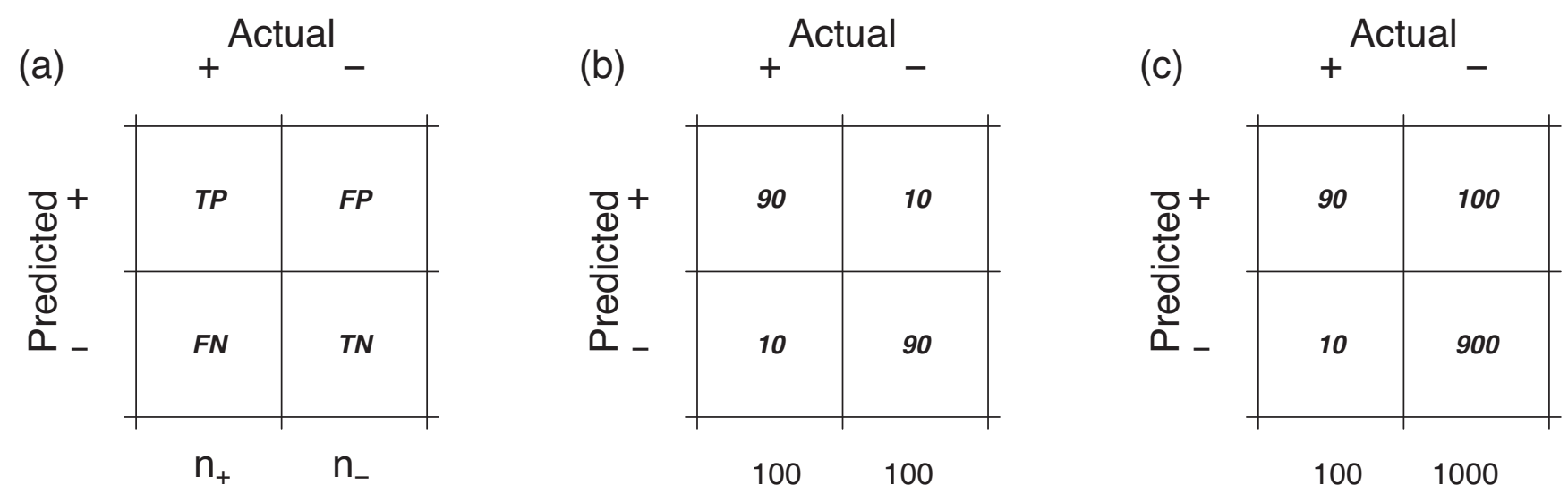

Figure 1. Cross-tabulation of actual and predicted classes, based on a specified threshold. (a) gives standard notation. (b) displays a numerical example where there are equal numbers of positive and negative objects. (c) displays a numerical example where there are ten times as many negative objects as there are positive objects. According to $a c c, a c c^{+}$, and $a c c^{-}$, the examples in (b) and (c) are equivalent in quality. According to prec, the example in (c) has much worse performance than that in (b).

funding applicant two, while classification suggests that applicants two and four are equally worthy. Classification may occur after the ranking task is complete, but ranking needs more information than that provided by classification alone. This article is primarily concerned with the task of ranking objects. Assessment for ranking performance requires extra investigations beyond classification performance. Two popular assessment measures are compared, along with their uncertainty under perturbation of data.

Consider a collection of $N$ objects with algorithm scores $S_{1}, S_{2}, \ldots, S_{N}$. Suppose these objects are classified by applying a threshold $t$. If the true membership classes for the objects could be determined, a natural check on the effectiveness of the classification task is a cross-tabulation according to predicted class and actual class, as shown in Figure 1(a). The counts $T P$ and $T N$ represent correct classifications and are referred to as the number of true positives and the number of true negatives. $F P$ is the count of objects that are classified as positive but are actually negative, i.e., the number of false positives. $F N$ is the number of false negatives. In any collection where class membership is static, the number of actual positives, $n_{+}$, does not change, and $T P+F N=n_{+}$. Similarly, $n_{-}=F P+T N$ is the number of actual negatives. A goal of classification is to maximize $T P+T N$ or maximize accuracy $(a c c)$, defined as $(T P+T N) / N$. This goal may be subdivided as maximizing accuracy-for-positive-objects $\left(\mathrm{acc}^{+}\right)$, defined as $T P / n_{+}$, and maximizing accuracy-for-negativeobjects $\left(\operatorname{acc}^{-}\right)$, defined as $T N / n_{-} . \operatorname{acc}^{+}$is commonly referred to as the true positive rate $(t p r)$ or sensitivity or recall. $a c c^{-}$is very commonly referred to as specificity or $1-$ the false positive rate $(f p r)$. Note that $a c c$ is a weighted average of $a c c^{+}$and $a c c^{-}: a c c=w \cdot a c c^{+}+$ $(1-w) \cdot a c c^{-}$where $w=n_{+} / N$ is the observed fraction of positive objects.

Accuracy, while desirable even for the task of ranking, can mask undesirable performance when one class has far more members than the other class; this is the situation of class imbalance or low prevalence. Figures 1(b) and 1(c) show two classifiers with high values of 0.9 for $a c c, a c c^{+}$, and $a c c^{-}$. But the classifier in Figure 1(c) is actually much worse than the one in Figure 1(b) in that 90 percent of the predicted positives are actually positive in Figure 1(b), while only 47 percent of the predicted positives are actually positive in Figure 1(c). This illustration demonstrates the usefulness of precision ( $\mathrm{prec}$ ) as a measure of assessment. Precision, also known as positive predictive value, is the fraction of predicted positives that are actually positive, and is obtained as $T P /(T P+F P)$. The classifiers in Figures $1(\mathrm{~b})$ and $1(\mathrm{c})$ have prec $=0.9$ and prec $=0.47$, respectively.

It is important to realize that $\mathrm{acc}^{+}, \mathrm{acc}^{-}$, and prec can change according to changing the threshold, because changing the threshold can then lead to different tables as in Figure 1(a). To stress this dependence, we use notation $\operatorname{acc}_{t}^{+}, a c c_{t}^{-}$, and $\operatorname{prec}_{t}$. Ideally, assessment for both classification and ranking should investigate the extent to which all of $a c c_{t}^{+}, a c c_{t}^{-}$, and prec $_{t}$ are large, for some ideal threshold or range of thresholds. The most popular approaches for assessment, however, unfortunately only focus on two of the three measures.

The receiver operating characteristic (ROC) curve focuses on $\mathrm{acc}^{+}$and $a c c^{-}$. It plots $1-\operatorname{acc}_{t}^{-}$as the abscissa and $a c c_{t}^{+}$as the ordinate, for different values of $t$. For largest $t$, the ROC curve starts at $(0,0)$. As $t$ decreases, the ROC curve is nondecreasing, ending at $(1,1)$ for the smallest $t$. The ideal location on the ROC curve is the 

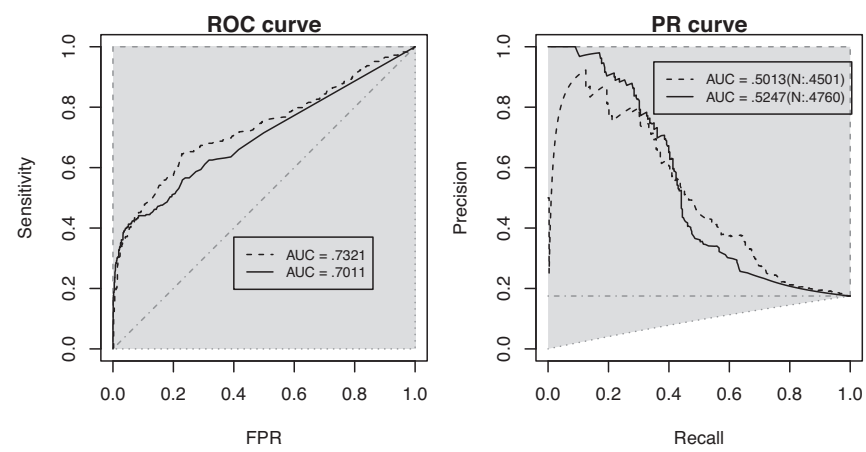

Figure 2. Estimated ROC and PR curves for the MAO dataset. Curves resulting from the random forest prediction algorithm are solid while curves from the logistic prediction algorithm are dashed and black. Shaded areas reflect the maximum and minimum possible curves, and the dot-dashed lines correspond to random guessing. Areas under each curve (AUC) are also shown. For the PR curves, normalized areas under the curve are preceded by "N:". Using $R O C$, logistic regression is better. Using $P R$, random forest is better. For ranking, random forest is better because three of the four largest logistic scores are assigned to inactive compounds while the 28 largest random forest scores are all assigned to active compounds.

point $(0,1)$, but in practice this is rarely achieved. There is a large body of literature on methods for determining optimal or near-optimal thresholds, all based on the idea of getting as close to $(0,1)$ as possible; see, for example, [15]. An alternative strategy (one that this article uses) is to avoid selecting a single threshold and instead assess the ROC curve over all thresholds by obtaining the area under the ROC curve, denoted ROC (note difference in font) hereafter. Ideal classification results in $R O C=1$, while random guessing results in $R O C=0.5$. The ROC curve and resulting $R O C$ have been extensively used and studied in many fields, including medical testing, artificial intelligence, machine learning, and banking. See, for example, [14], [8], and [13].

Because it does not monitor prec, which can be appallingly low when classes are imbalanced (as implied in Figure 1), the ROC curve has lost prominence in the presence of imbalance. Its replacement, the precision-recall (PR) curve, plots $\operatorname{acc}_{t}^{+}$as the abscissa and $p r e c_{t}$ as the ordinate, for different values of $t$. The ideal location on the PR curve is the point $(1,1)$. Area under the PR curve, denoted $P R$ hereafter, has become a popular means of summary. However, while $R O C$ can take values from zero to one for $N$ sufficiently large and irrespective of class imbalance, $P R$ cannot ([2], [3], [12]). PR curves are more affected than ROC curves by characteristics of the underlying population of objects to be ranked. Specifically, PR curves created from populations with balanced classes (i.e., prevalence near 0.5) will look very different from PR curves created from populations with class imbalance. PR curves directly reflect the degree of imbalance in the data in two primary ways ([6], [1],[10]). First, as $t$ approaches its lower limit, $\left(a c c_{t}^{+}, p r e c_{t}\right)$ often approaches $(1, w)$, where recall that $w$ is the fraction of objects that are positive. Second, the PR curve is bounded below, thus preventing $P R$ from taking the value zero. A consequence is that $R O C$ values may be directly compared across different datasets, but $P R$ values must be normalized before being compared across different datasets. A reasonable normalization is $\left(P R-P R_{\text {min }}\right) /\left(1-P R_{\text {min }}\right)$, where $P R_{\text {min }}$ is the smallest possible value of $P R$ and it has a closed-form expression ([2], [3], [12]). If, however, different datasets are forced to have the same frequencies of positive and negative objects, then $P R$ many be compared without normalization.

While the PR curve gained popularity to address issues regarding class imbalance, it is also very useful for assessing ranking algorithms. Ranking algorithms produce an ordered arrangement of objects where objects earlier in the ranking are expected to have a greater chance of being positive. By its very definition, prec rewards this type of ranking so that only effective ranking algorithms will have high PR curves.

\section{Monoamine Oxidase Inhibition}

[4] was one of the first papers to make a pharmaceuticalquality drug discovery dataset publicly available. A collection of 1646 compounds were tested for their monoamine oxidase (MAO) inhibition ability. MAO inhibitors gained popularity in the 1950s for treatment of depression. Today they are used to treat Parkinson's disease [17]. Compounds were placed in four categories: 1358 have potency value zero (inactive, i.e., negative); 114 have potency value one (mildly active); 86 have potency value two (moderately active); and 88 have the highest potency value of three (highly active). Many papers studied these groups and found that the high potency group is dominated by 

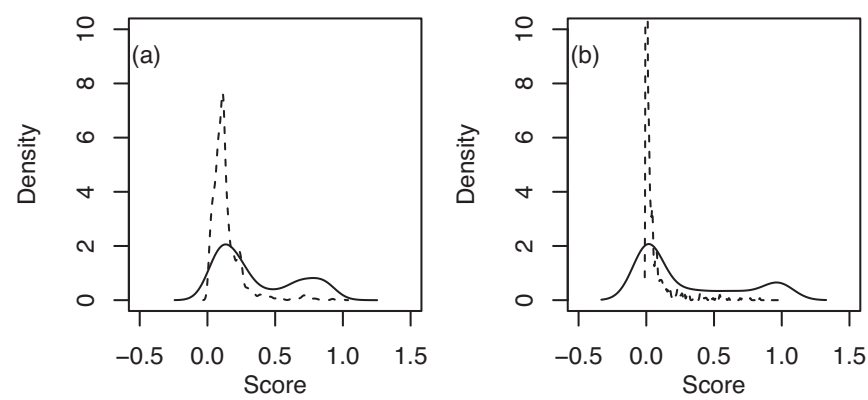

Figure 3. Estimated density curves of prediction scores for the MAO dataset: (a) for the logistic model and (b) for the random forest model. Curves corresponding to prediction score for the negative class are dashed while curves for the positive class are solid.

two mechanisms that are relatively easy to uncover [20]. On the other hand, the mild and moderate potency groups yield no clear structure or pattern. As a result, prediction algorithms tend to have extreme difficulty identifying the mild and moderate potency groups. This extra difficulty is a major motivation for selecting this dataset for illustrating subtleties between ranking and classification.

To convert the MAO data to a binary problem, the three potency groups are combined to give a single class of actives or positives. This results in $w=0.175$ as the fraction of positive objects. While this level of imbalance is not as extreme as commonly seen in drug discovery prediction tasks (where $w$ can easily be 0.001 or smaller), it is imbalanced enough to be noticeable. Analysis begins by developing prediction models that use a compound's chemical structure to predict activity. Many approaches are possible, but here I present output from just two models: logistic regression and random forest. Both logistic regression and random forest provide scores that represent the estimated probability that a compound (given its particular chemical structure) is active.

While some authors use the same data to build their prediction models and to create their ROC and/or PR curves, I believe this practice gives an unrealistically optimistic view of the effectiveness of the model, in much the same way that in-sample measures are frowned upon in model selection. Instead, I conduct stratified five-fold cross validation to obtain scores for both the logistic and random forest models. In other words, five sets of (training dataset, test dataset) pairs are created such that each compound appears in exactly one test dataset, and all training-test pairs have approximately the same degree of imbalance. A logistic or random forest model is built using data in a particular training set, then this model is applied to make predictions on the associated test set. The process is repeated for all training-test pairs. The overall result is a single prediction (i.e., score) for each compound, where that compound was not used in estimating the model that provided its prediction. (The idea is similar to pre-validation as described in [18].) These are the scores used to create estimated ROC and PR curves as shown in Figure 2. All computing was done in R [16], and ROC and PR curves and their corresponding $R O C$ and $P R$ were produced using package PRROC [12], [9].

According to the ROC curve, the logistic model is better with $R O C=0.7321$, compared to $R O C=0.7011$ for the random forest model. But a closer look at these curves reveal that the models appear equivalent up to a false positive rate of around 0.1 ( $\mathrm{CCC}^{-}$around 0.9 ), yielding sensitivity $\left(a c c^{+}\right)$over 0.4 . Pharmaceutical companies have compound libraries that commonly contain over one million compounds. If the fraction of truly active compounds is tiny, a false positive rate of just 0.1 could result in over 100,000 compounds being predicted as active when they are not. Because compounds predicted as active early in the ranking receive additional testing at great expense, this would be a big waste of resources. For this reason, drug discovery applications are interested in regions of very low false positive rates. The fact that the logistic model has a much higher ROC curve for higher false positive rates is of no practical value.

According to the PR curve, the random forest model is better with unnormalized $P R=0.5247$ and normalized $P R=0.4760$, compared to 0.5013 and 0.4501 for the logistic model. First, the fact that $P R$ and $R O C$ can give different orderings, even though PR-space and ROC-space have duality, is well known [7]. Second, because prec directly addresses the effect of ranking (while $\operatorname{acc}^{+}$and $\mathrm{acc}^{-}$do not), the PR curve is better able to show that three of the four largest logistic scores are assigned to inactive compounds while the 28 largest random forest scores are assigned to active compounds. For ranking algorithms, it is critical that objects with the largest predicted scores actually turn out to be positive, because these are the objects that will be acted on first; correct predictions later in the ranking have less relevance. The logistic PR curve eventually exceeds the random forest PR curve, but the damage from poor early rankings cannot be overcome. 
For this dataset, the random forest $P R$ is better than the logistic $P R$, but is this difference significant and important? If the MAO dataset were slightly perturbed, e.g., from swapping a few of the investigated compounds, would we reach the same conclusion? What if I repeated my fivefold cross validation but used a different way of assigning compounds to the training-test pairs? The bottomline is that this entire activity is reasonably viewed as an experiment, and we have observed one of many possible outcomes. Any decision about the quality of the logistic model versus the random forest model must address uncertainties that have not yet been explored.

To begin this exploration, I first attempt to estimate the underlying score distributions (see the following section for more details). Figure 3(a) shows estimated score densities for the logistic model, while Figure 3(b) shows estimated score densities for the random forest model. In both cases, the negatives have an estimated density with scores close to zero, but a long right tail results in scores that can be very close to one. The positives have a bimodal density that appears to be reasonably modeled as a mixture of normals. One mode coexists with the mode for the negatives; this mostly represents the 200 active compounds from the mild and moderate potency groups that we expected would be difficult to identify. The other mode is far away and represents the high potency group that we expected would be relatively easy to find. The best algorithms are able to completely separate the $F_{-}$and $F_{+}$distributions. Clearly, neither the logistic nor the random forest algorithms have achieved separation. In fact, separation was not expected for the MAO data because of previous studies explaining extreme difficulty of identifying the mild and moderate potency groups.

\section{Probabilistic Definitions of ROC and PR Curves}

To formalize the probabilistic basis of ROC and PR curves, we consider $S$, the score produced by a prediction algorithm, to be a random variable. An object is predicted as positive if $S>t$ and negative otherwise, for some specified threshold $t$. Conditioned on the object being truly positive, the distribution function for $S$ is given as $F_{+}(\cdot)$. Similarly, $F_{-}(\cdot)$ is the distribution function for $S$ conditioned on the object being truly negative. Let $\pi_{+}$denote the true probability of membership in the positive class. [6] and [10] are good references for additional details of what follows.

The ROC curve plots $\left(x_{\mathrm{ROC}}, y_{\mathrm{ROC}}\right)$, where

$$
\begin{aligned}
& x_{\mathrm{ROC}}=\operatorname{Pr}(S>t \mid-)=1-F_{-}(t) \\
& y_{\mathrm{ROC}}=\operatorname{Pr}(S>t \mid+)=1-F_{+}(t)
\end{aligned}
$$

for all $t \in \mathbb{R}$, with functional representation as

$$
y_{\mathrm{ROC}}\left(x_{\mathrm{ROC}}\right)=1-F_{+}\left(F_{-}^{-1}\left(1-x_{\mathrm{ROC}}\right)\right), \quad 0<x_{\mathrm{ROC}}<1,
$$

where $F_{-}^{-1}(\cdot)$ is a generalized inverse of distribution function $F_{-}(\cdot)$.

The PR curve plots $\left(x_{\mathrm{PR}}, y_{\mathrm{PR}}\right)$, where

$$
\begin{array}{ll}
x_{\mathrm{PR}}=\operatorname{Pr}(S>t \mid+) & =y_{\mathrm{ROC}} \\
y_{\mathrm{PR}}=\operatorname{Pr}(+\mid S>t) & =\frac{\pi_{+}}{\pi_{+}+\left(1-\pi_{+}\right)\left(x_{\mathrm{ROC}} / y_{\mathrm{ROC}}\right)}
\end{array}
$$

for all $t \in \mathbb{R}$, with functional representation as

$$
y_{\mathrm{PR}}\left(x_{\mathrm{PR}}\right)=\frac{\pi_{+}}{\pi_{+}+\left(1-\pi_{+}\right)\left[1-F_{-}\left(F_{+}^{-1}\left(1-x_{\mathrm{PR}}\right)\right)\right] / x_{\mathrm{PR}}},
$$

Of course, one never knows $F_{+}(\cdot), F_{-}(\cdot)$, or $\pi_{+}$, so they all must be estimated using data. Suppose we observe independent random samples of scores $\left\{S_{i}^{+} ; i=1, \ldots, n_{+}\right\}$ from the positive class and $\left\{S_{i}^{-} ; i=1, \ldots, n_{-}\right\}$from the negative class. This data can be used to produce estimates of $\pi_{+}\left(\right.$call it $\left.\hat{\pi}_{+}=n_{+} /\left(n_{+}+n_{-}\right)\right), F_{+}\left(\right.$call it $\left.\hat{F}_{+}\right)$, and $F_{-}$(call it $\hat{F}_{-}$) that may be directly inserted into equations (1) and (2) to give estimated ROC and PR curves. Varying levels of assumptions may be applied to obtain $\hat{F}_{+}$ and $\hat{F}_{-}$, ranging from fully parametric, to semiparametric, to nonparametric ([14], [13]). For example, $\hat{F}_{+}$and $\hat{F}_{-}$ could denote the nonparametric class-level empirical distribution functions, i.e., $\hat{F}_{+}(t)=\frac{1}{n_{+}} \sum_{i=1}^{n_{+}} I\left(S_{i}^{+} \leq t\right)$ and $\hat{F}_{-}(t)=\frac{1}{n_{-}} \sum_{i=1}^{n_{-}} I\left(S_{i}^{-} \leq t\right)$, where $I(\cdot)$ is the indicator function. Other, simpler but often less desirable, estimation strategies lead to the formulas given in the introduction. To see this, it may be helpful to note that the terms $T P, F P, F N$, and $T N$ can all be represented using notation from this section:

$$
\begin{array}{ll}
T P=\sum_{i=1}^{n_{+}} I\left(S_{i}^{+}>t\right) & F P=\sum_{i=1}^{n_{-}} I\left(S_{i}^{-}>t\right) \\
F N=\sum_{i=1}^{n_{+}} I\left(S_{i}^{+} \leq t\right) & T N=\sum_{i=1}^{n_{-}} I\left(S_{i}^{-} \leq t\right) .
\end{array}
$$

To visualize the impact of different probabilistic models on ROC and PR curves, and to help understand difficulties that can arise from bimodal distributions as evidenced in the MAO example, I consider three population-level algorithms. Cases are fully described in Table 1. Each algorithm consists of distributions $F_{-}(\cdot)$ and $F_{+}(\cdot)$, and in all cases $\pi_{+}$is set to 0.175 as observed in the MAO example. $F_{-}(\cdot)$ is always normally distributed with mean 0.3 and standard deviation 0.05 . The cases differ only in terms of 


\begin{tabular}{c|l|c|c} 
Case & $F_{+}$distribution: mixture of normals with 0.5 mixing proportion & $P R$ & $R O C$ \\
\hline \hline 1 & $\begin{array}{l}\text { component 1: mean is 0.3, standard deviation is } 0.25 \\
\text { component 2: mean is 0.7, standard deviation is 0.05 }\end{array}$ & 0.749 & 0.750 \\
\hline 2 & $\begin{array}{l}\text { component 1: mean is 0.3, standard deviation is 0.05 } \\
\text { component 2: mean is 0.7, standard deviation is 0.05 }\end{array}$ & 0.650 & 0.750 \\
\hline 3 & $\begin{array}{l}\text { component 1: mean is 0.3, standard deviation is 0.005 } \\
\text { component 2: mean is 0.7, standard deviation is 0.05 }\end{array}$ & 0.616 & 0.750 \\
\hline
\end{tabular}

Table 1. Three cases of population-level algorithms. Objects in the negative class have scores that follow a normal distribution with mean 0.3 and standard deviation 0.05 . Objects in the positive class have scores that follow a two-component mixture of normal distributions, with details of the components given in the table. $P R$ and $R O C$ values are also given for each algorithm. While $P R$ changes across different cases, $R O C$ remains the same.
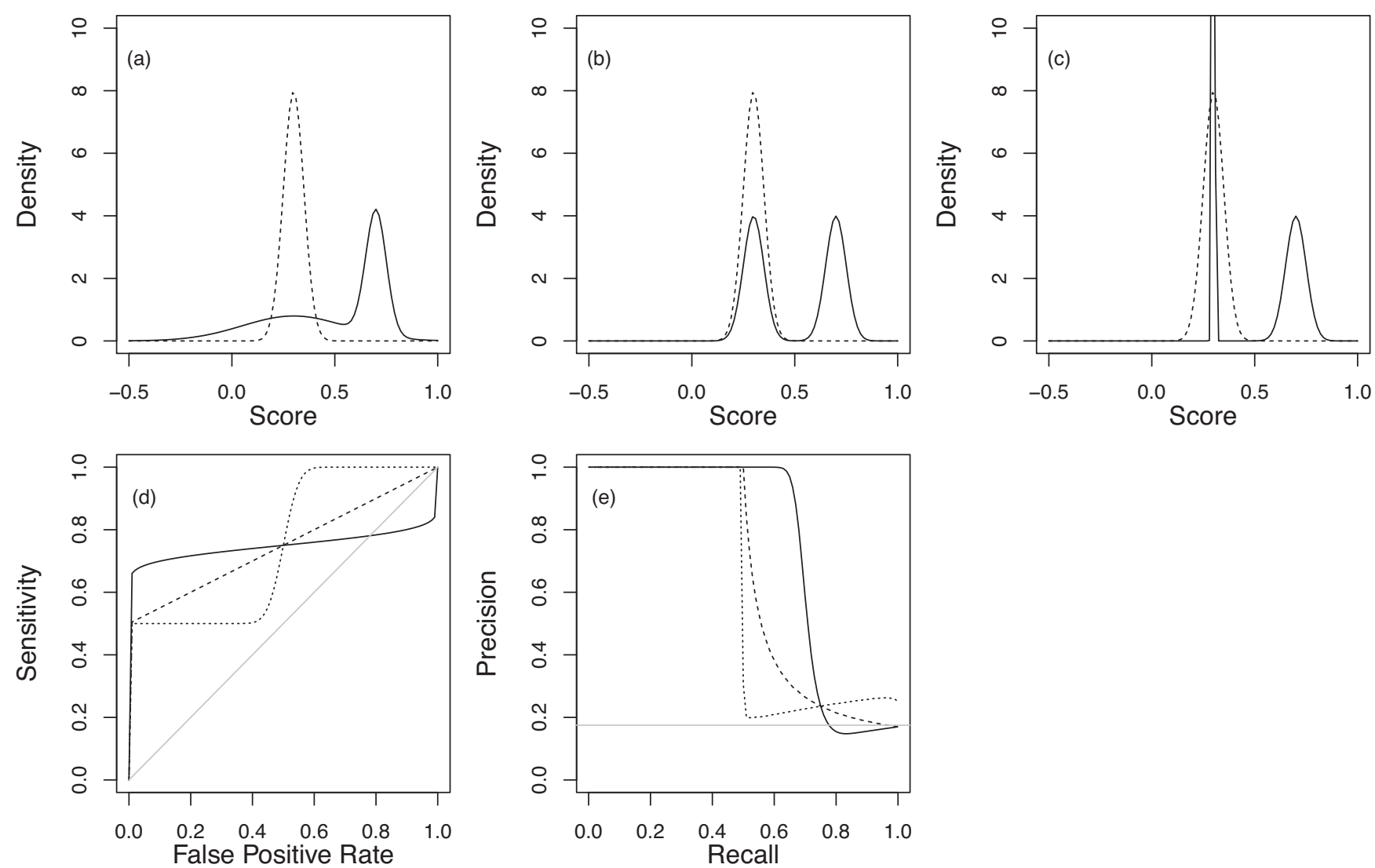

Figure 4. Densities, ROC curves, and PR curves for three cases of population-level algorithms. (a), (b), (c) display the densities corresponding to negative objects as dashed curves, and densities for positive objects as solid curves; (a) is Case 1, (b) is Case 2, and (c) is Case 3 as described in Table 1. (d) displays ROC curves for all three cases: Case 1 is solid, Case 2 is dashed, Case 3 is dotted, and the grey curve depicts random guessing. (e) displays PR curves for all three cases: Case 1 is solid, Case 2 is dashed, Case 3 is dotted, and the grey curve depicts random guessing. $R O C=0.75$ for all three cases, but $P R$ correctly assesses Case 1 as best able to rank objects $(P R=0.749)$, and Case 3 as worst $(P R=0.616)$. 
$F_{+}(\cdot)$, which is always a two-component mixture of normals where the component far from the negative distribution $F_{-}(\cdot)$ always has mean 0.7 and standard deviation 0.05 . The other component of $F_{+}(\cdot)$ has the same mean as $F_{-}(\cdot)$, namely 0.3 , but its standard deviation varies from a high of 0.25 , to 0.05 , to 0.005 . Densities for all cases are shown in Figures 4(a), 4(b), and 4(c).

The mixing proportion of 0.5 for distribution $F_{+}$implies that all three algorithms will quickly identify 50 percent of the positives, namely those coming from the rightmost component of $F_{+}$. The ROC curves and PR curves of Figures 4(d) and 4(e) show this as all curves overlap for recall (sensitivity) values between zero and 0.5. The curves separate for other values of recall. Case 1, with the largest standard deviation for the leftmost component of $F_{+}$, perfectly identifies an additional 12 percent of positives before it struggles significantly, even dropping worse than random; it has the largest $P R$. Case 3, with the smallest standard deviation for the leftmost component of $F_{+}$, is exquisitely poor after identifying the first 50 percent of positives. It creates over 40 percent of false positives before restarting to find true positives at a uniform rate until all are found. Case 3 has the smallest $P R$. Case 2 finds the second half of positives at a uniform rate after finding the first half without errors. In other words, Case 1 does a better job of finding the actives early, Case 3 finds the actives late, and Case 2 finds the actives uniformly. [19] argue that algorithms designed for the "early recognition" (aka ranking) problem should be able to properly distinguish these cases. $P R$ does an excellent job of assessing these algorithms. $R O C$, on the other hand, remains exactly the same (0.75) for all three cases. $R O C$ is not able to make a distinction. The ROC curves clearly differ, and if focus is limited to early in the curves, it is clear that the proper ordering of algorithms is obtained. But this information is lost when area under the entire curve is calculated as the primary measure of performance, as might become necessary when comparing multiple algorithms.

\section{Investigating Uncertainty}

When data is used to obtain the PR and ROC curves and their associated $P R$ and $R O C$ values, effort must be expended to understand the uncertainty or variability associated with these estimates. A large body of literature exists on statistical inference for $R O C$; see, for example, [14], [19], [13]. The literature is scant regarding inference on $P R$, but findings generally suggest that bootstrap sampling is the low-risk method of choice ([2], [3]). I will use bootstrap sampling to explore both $P R$ and $R O C$.

Consider the scenario presented in the MAO example, where there are two competing algorithms. "Which is better?" may well be answered by the difference between their areas under the curves, namely $P R_{2}-P R_{1}$ or $R O C_{2}-$

\begin{tabular}{lc|c|c|c|} 
& & \multicolumn{3}{c}{ Case for } \\
& \multicolumn{3}{c}{ Algorithm 2 } \\
\cline { 3 - 5 } Case for & 1 & A & B & C \\
\cline { 2 - 4 } Algorithm 1 & 2 & & D & E \\
\cline { 2 - 5 } & 3 & & & F \\
\cline { 3 - 4 } & & &
\end{tabular}

Table 2. Six scenarios, A-F, for algorithmic comparison. Each scenario describes two algorithms, with the eventual goal of determining which algorithm is better. For example, Scenario B has algorithm 1 defined according to Case 1 as given in Table 1, and algorithm 2 defined according to Case 2. In Scenarios A, D, and F, the two algorithms have the exact same probabilistic settings, so no differences are expected.

$R O C_{1}$. Algorithm 1 is better if $P R_{2}-P R_{1}<0$, so it makes sense to obtain a confidence interval for $P R_{2}-P R_{1}$, then the degree to which the interval contains negative values determines our answer. By now, skepticism should be high in your mind regarding the utility of $R O C_{2}-R O C_{1}$ for assessing ranking performances, so we do not expect the confidence interval to the useful.

Six scenarios are studied, as outlined in Table 2. All scenarios apply distributional settings for both algorithms from among Cases 1, 2, and 3 of Table 1. Scenarios A, $\mathrm{D}$, and $\mathrm{F}$ are null in that both algorithms have exactly the same probabilistic settings, so there should be no differences in values of $P R$; the confidence intervals are expected to include zero. Scenarios B, C, and E are non-null and differences are expected. The differences for $\mathrm{C}$ are expected to be largest (approximately 0.616 $-0.749=-0.133$ ), followed by B (approximately $0.650-0.749=-0.099$ ), then E (approximately $0.616-0.650=-0.034$ ).

Bootstrap sampling reduces the need for assumptions that are often tenuous at best. New pseudo-samples are created from the original sample by sampling with replacement. My sampling was stratified to maintain the level of imbalance. For each simulation replicate within each Scenario A to F, 1000 bootstrap samples are obtained and these samples are used to determine a percentile confidence interval. $\mathrm{R}$ package boot [5] was used for bootstrapping. Figure 5(a) displays 95 percent confidence intervals for $P R_{2}-P R_{1}$ from 50 simulation replicates for all of Scenarios A-F. Consistent with expectations, the confidence intervals for Scenario $\mathrm{C}$ are most negative, followed by Scenario $\mathrm{B}$ then $\mathrm{E}$. It was surprising to me that the confidence intervals for Scenarios A, D, and F do not overwhelmingly include zero. They are close to zero, so may be treated as not practically different from zero, but they bounce around, sometimes above other times below zero. Increasing the 

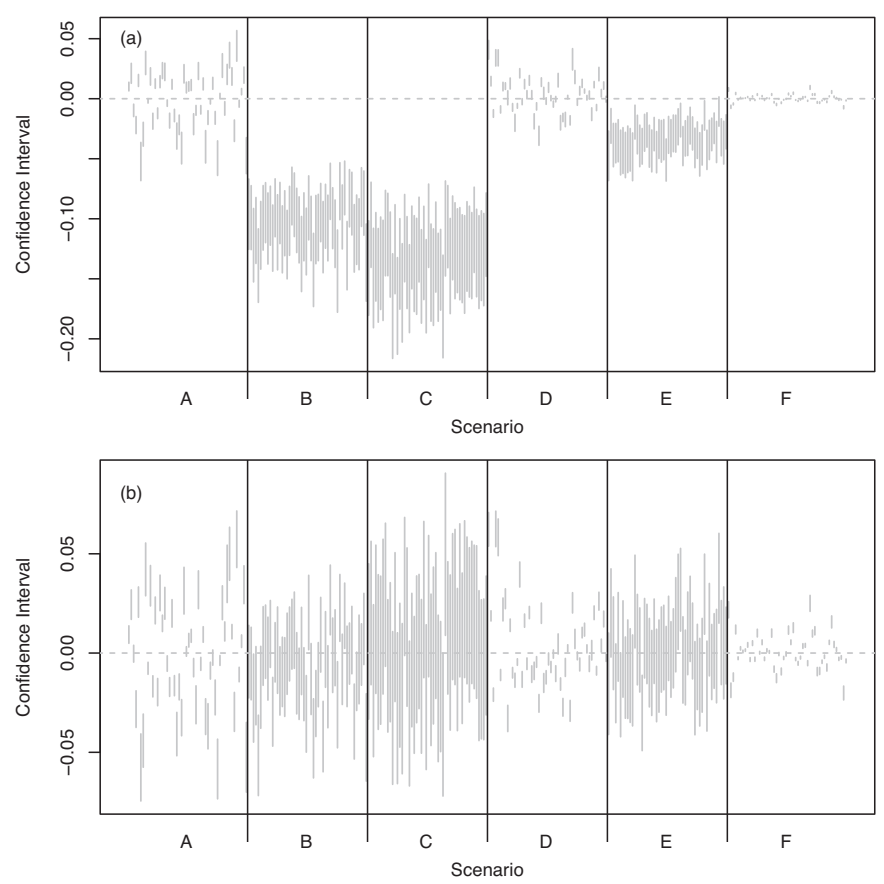

Figure 5. Ninety-five percent confidence intervals for $P R_{2}-P R_{1}$ (plot (a)) and $R O C_{2}-R O C_{1}$ (plot (b)) from the six scenarios of Table 2 . For each scenario and each independently obtained sample, a bootstrap percentile confidence interval is shown where upper and lower endpoints are connected by grey lines. For scenarios where both algorithms are probabilistically equivalent (i.e., A, D, and F), one expects approximately 95 percent of the intervals to include zero. Results are shown based on 1000 bootstrap samples for each of 50 independent samples generated from each scenario.

number of bootstrap samples had no impact. Another observation is that the uncertainty level (wide intervals mean greater uncertainty) of the estimated $P R_{2}-P R_{1}$ decreases as the estimate itself gets closer to zero, and more rapidly so if the individual components $\left(P R_{2}, P R_{1}\right)$ are small.

Figure 5(b) displays 95 percent confidence intervals for $R O C_{2}-R O C_{1}$ from 50 simulation replicates for Scenarios A-F. Recall that $R O C$ for Cases $1-3$ are all 0.75 , so no differences are expected. Confidence intervals for Scenarios $\mathrm{B}, \mathrm{C}$, and $\mathrm{E}$ now mostly cross zero, unlike in Figure $5(\mathrm{a})$. It is surprising that Scenarios $\mathrm{A}, \mathrm{D}$, and $\mathrm{F}$ behave similar to how they are in Figure 5(a), with confidence intervals hardly ever crossing zero but bouncing above or below zero.

\section{Summary}

The PR and ROC curves, and their corresponding area under the curve measures, were compared as assessment criteria for effective ranking by prediction algorithms for the two-class problem. $R O C$ is shown to be particularly deficient while $P R$ is quite effective. A real dataset revealed a bimodal score distribution for the positive class, and this resulted in problems with discrimination between scores for the different classes of objects. A simulation study revealed unusual patterns in confidence intervals for differences $P R_{2}-P R_{1}$ and $R O C_{2}-R O C_{1}$ when algorithms 1 and 2 are probabilistically equivalent. Further studies are needed to understand these uncertainties.

ACKNOWLEDGMENT. I thank the reviewers for very useful comments that led to an improved manuscript.

\section{References}

[1] Kendrick Boyd, Vítor Santos Costa, Jesse Davis, and C. David Page, Unachievable region in precision-recall space and its effect on empirical evaluation, Proceedings of the 29th international conference on machine learning, 2012, pp. 1619-1626.

[2] Kendrick Boyd, Kevin H. Eng, and C. David Page, Area under the precision-recall curve: Point estimates and confidence intervals: Lecture notes in computer science, vol 8190, Machine learning and knowledge discovery in databases - european conference, ECML PKDD 2013, prague, czech republic, september 23-27, 2013, proceedings, part III, 2013, pp. 451466.

[3] Kendrick Boyd, Kevin H. Eng, and C. David Page, Erratum: Area under the precision-recall curve: Point estimates and confidence intervals, Machine learning and knowledge discovery in databases - european conference, ECML PKDD 2013, prague, czech republic, september 23-27, 2013, proceedings, part III, 2013.

[4] Robert D. Brown and Yvonne C. Martin, Use of structureactivity data to compare structure-based clustering methods and descriptors for use in compound selection, Journal of Chemical Information and Computer Sciences 36 (1996), 572-584.

[5] Angelo Canty and Brian Ripley, boot: Bootstrap R (S-plus) functions, 2016. R package version 1.3-18.

[6] Stéphan Clémençon and Nicolas Vayatis, Nonparametric estimation of the precision-recall curve, Proceedings of the 26th international conference on machine learning - ICML '09, 2009, pp. 185-192.

[7] Jesse Davis and Mark Goadrich, The relationship between precision-recall and ROC curves, Proceedings of the 23rd international conference on machine learning - ICML '06, 2006, pp. 233-240.

[8] Tom Fawcett, An introduction to ROC analysis, Pattern Recognition Letters 27 (2006), no. 8, 861-874.

[9] Jan Grau, Ivo Grosse, and Jens Keilwagen, PRROC: computing and visualizing precision-recall and receiver operating characteristic curves in R, Bioinformatics 31 (2015), no. 15, 2595-2597.

[10] Jacqueline M. Hughes-Oliver, Population and empirical PR curves for assessment of ranking algorithms, 2018. submitted. 
[11] Gareth James, Daniela Witten, Trevor Hastie, and Robert Tibshirani, An introduction to statistical learning, with applications in R, Springer Texts in Statistics, vol. 103, Springer, New York, 2013. MR3100153

[12] Jens Keilwagen, Ivo Grosse, and Jan Grau, Area under precision-recall curves for weighted and unweighted data, PLOS ONE 9 (2014), no. 3. e92209.

[13] Wojtek J. Krzanowski and David J. Hand, ROC curves for continuous data, Monographs on Statistics and Applied Probability, vol. 111, CRC Press, Boca Raton, FL, 2009. MR2522628

[14] Margaret Sullivan Pepe, The statistical evaluation of medical tests for classification and prediction, Oxford Statistical Science Series, vol. 28, Oxford University Press, Oxford, 2003. MR2260483

[15] Foster Provost and Tom Fawcett, Robust classification for imprecise environments, Machine Learning 42 (2001Mar), no. 3, 203-231.

[16] R Core Team, $R$ : A language and environment for statistical computing, R Foundation for Statistical Computing, Vienna, Austria, 2016.

[17] Peter Riederer and Gerd Laux, MAO-inhibitors in parkinson's disease, Experimental Neurobiology 20 (20113), 1-17.

[18] Robert J Tibshirani and Brad Efron Pre-validation and inference in microarrays, Statistical Applications in Genetics and Molecular Biology 1 (2002), no. 1.

[19] Jean-François Truchon and Christopher I. Bayly Evaluating virtual screening methods: Good and bad metrics for the 'early recognition' problem, Journal of Chemical Information and Modeling 47 (20073), no. 2, 488 -508.

[20] Ke Zhang, Jacqueline M. Hughes-Oliver, and S. Stanley Young Analysis of high-dimensional structureactivity screening datasets using the optimal bit string tree, Technometrics 55 (2013), no. 2, 161-173, DOI 10.1080/00401706.2012.760489. MR3176517

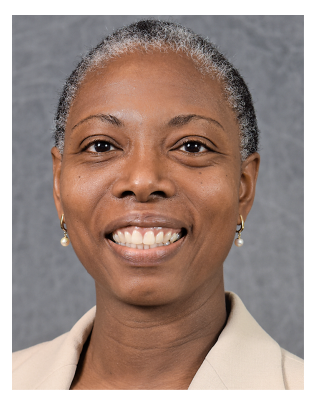

Jacqueline M. Hughes-Oliver

Credits

Article photos are courtesy of Jacqueline M. Hughes-Oliver. Author photo is courtesy of Becky Kirkland. 